\title{
Past and Present Terminology for the Retinal and Choroidal Structures in Optical Coherence Tomography
}

Burak Turgut

FIrat University, School of Medicine, Department of Ophthalmology, Elazig, Turkey

DOl: https://doi.org/10.17925/EOR.2017.11.01.59

S pectral domain optical coherence tomography (SD-OCT) is a non-invasive imaging method which is used in the diagnosis and followup of various macular diseases. SD-OCT provides detailed imaging of the retina. However, it has also been used to evaluate the choroidal layers. There are many publications on the OCT terminology, the definition and classification of retinal and choroidal structures including lines, bands and zones described in OCT. The aim of this review is to provide an overview of the literature on the past and present terminology for the retinal and choroidal structures seen in SD-OCT. To knOw OCT terminology will provide to be understanding better the pathogenesis of these diseases and the effects of therapeutic applications for these.

\section{Keywords}

Optical coherence tomography, terminology, international nomenclature

Disclosure: Burak Turgut has nothing to disclose with any trade or device in relation to this article. No funding was received for the publication of this article. This study involves a review of the literature and did not involve any studies with human or animal subjects performed by any of the authors.

Authorship: All named authors meet the International Committee of Medical Journal Editors (ICMJE) criteria for authorship of this manuscript, take responsibility for the integrity of the work as a whole, and have given final approval to the version to be published.

open Access: This article is published under the Creative Commons Attribution Noncommercial License which permits any non-commercial use, distribution, adaptation and reproduction provided the original author(s) and source are given appropriate credit.

Received: 20 March 2017

Accepted: 3 June 2017

Citation: European Ophthalmic Review,

2017;11(1):59-61

Corresponding Author: Burak Turgut, Associate

Professor of Ophthalmology, Firat University, Faculty of

Medicine, Department of Ophthalmology, 23119, Elazig,

Turkey. E: drburakturgut@gmail.com
Spectral domain optical coherence tomography (SD-OCT) is a very useful non-invasive imaging method which is used in the diagnosis and follow-up of various retinal and choroidal diseases involving macular region. ${ }^{1-3}$ In 2014, at International Nomenclature OCT (INOCT) Panel, Staurenghi et al. ${ }^{4}$ proposed a nomenclature system for normal anatomic landmarks in SD-OCT and it has been reached a consensus regarding the most proper terminology and normal anatomic landmarks in the posterior segment SD-OCT. In addition, it has been recommended as the standardised nomenclature for use in the future publication in the panel. Currently, this terminology has been commonly used by some ophthalmologists, especially retina specialists. ${ }^{4}$

In this review, based on the literature, it has been aimed to provide an overview of the definition and classification of the retinal and choroidal structures seen in SD-OCT, and to propose the standard nomenclature and terminology for these structures.

To know the INOCT, OCT terminology and nomenclature of retinal and choroidal layers, the landmarks, zones, bands and lines described in OCT in healthy eyes will also provide to be understanding better the pathogenesis of various macular diseases and the effects of therapeutic applications for these.

\section{The anatomical/histologic landmarks in current OCT literature and INOCT}

In an SD-OCT scan of a normal eye, the high reflectivity signals come from the retinal nerve fibre layer (INOCT, Zone 3), outer plexiform layer (OPL) (INOCT, Zone 7), inner plexiform layer (INOCT, Zone 5), internal limiting membrane (ILM), ellipsoid zone (EZ) of photoreceptors (INOCT, Zone 11), interdigitation zone (IZ) of photoreceptors (INOCT, Zone 13) and retina pigment epithelium (RPE)-Bruch's membrane (BM) complex (INOCT, Zone 14). The low reflectivity signals belong to the nuclear layers, ganglion cell layer, Henle's nerve fibre layer (HFL), myoid zone (MZ) of the photoreceptors (INOCT, Zone 10), and outer segments (OSS) of the photoreceptors (INOCT, Zone 12). The vitreous gel is not visible in OCT imaging of the healthy subjects because it is optically clear and transparent, and it seems black colour in OCT scans. However, posterior cortical vitreous (INOCT, Zone 1) can seem mildly hyper-reflective in the high-resolution OCT images in some healthy subjects (Figure 1). ${ }^{4-7}$

The external limiting membrane (ELM) (INOCT, Zone 9) is located at the boundary between the nuclei and the inner segments (ISs) of the photoreceptors and corresponds to the multiple junctional complexes between Müller cells and the photoreceptors. ${ }^{4-7}$

The outer (dendritic) plexiform layer (OPL) (INOCT, Zone 7) is the hyper-reflective layer that corresponds with the synapses between cone pedicles and rod spherules with dendrites of horizontal and bipolar cells. ${ }^{4-7}$ 
Figure 1: EDI-OCT image demonstrating INOCT zones, and layers from a healthy subject.

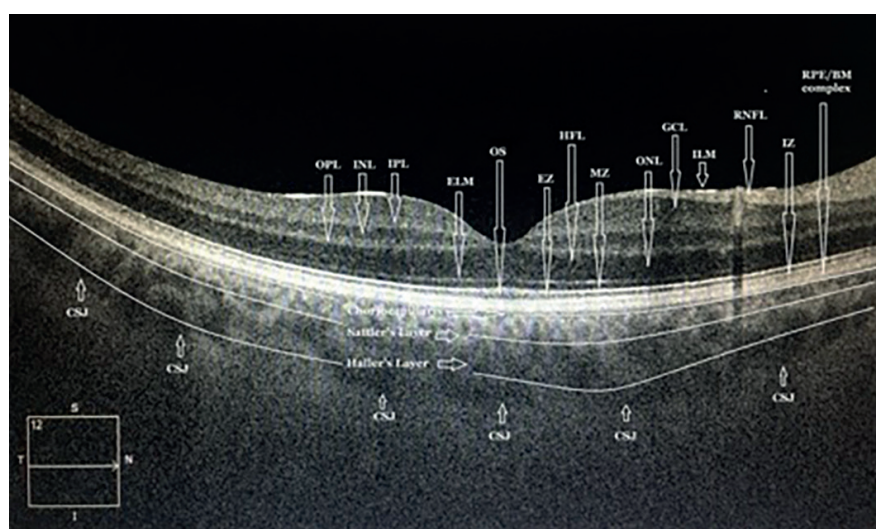

$B M=$ Bruch's membrane; $C S J=$ choroidal-scleral junction; $E D I=$ enhanced depth imaging; $E L M=$ external limiting membrane; $E Z$ = ellipsoid zone; $G C L$ = ganglion cell layer: $H F L=$ Henle's fibre laver; $I L M=$ internal limiting membrane; $I N L=$ inner nuclear layer; $I N O C T=$ International Nomenclature OCT; IPL = inner plexiform layer; $I Z=$ interdigitation zone; $\mathrm{MZ}$ = myoid zone; OCT = optical coherence tomography; ONL = outer nuclear layer; $O P L=$ outer plexiform layer; $O S=$ outer segments; $R N F L=$ retinal nerve fibre layer; RPE = retina pigment epithelium.

The outer nuclear layer (ONL) and HFL (INOCT, Zone 8) is the hyporeflective zone between ELM and the OPL was previously attributed to the ONL., ${ }^{3,8-12} \mathrm{HFL}$, composed of the axons of photoreceptors can be seen to contribute to the inner region of Zone 8 . HFL is not present in the mid-peripheral/peripheral retina. HFL is exactly a part of the OPL which is observed as hyper-reflective in OCT. However, it seems hypo-reflective because the fibres in HFL obliquely course in retinal structure. Thus, ONL is observed thicker than actual. In addition, recent studies showed that HFL could be seen as hyper-reflective zone if the beam of OCT can be directed to the retina. $4,13,14$

In retinal histology, ISs of photoreceptors are divided into two parts: myoid IS and ellipsoid IS. Myoid IS is close to the ELM while ellipsoid IS is close to the OSs of photoreceptors. ${ }^{8-9}$

The MZ (INOCT, Zone 10) is the hypo-reflective region and located between ELM and EZ, and it is close to the ELM. However, MZ has not been recognised as a distinct OCT retinal layer in the literature. MZ includes ribosomes, endoplasmic reticulum, Golgi bodies and rare mitochondria. It has been considered that this zone is corresponding to the myoid portion of the ISS of photoreceptors and that the hyporeflectivity of $\mathrm{MZ}$ is due to the lower packing density of mitochondria in this zone compared to the $\mathrm{EZ} \cdot{ }^{4-7,10-12}$

The EZ (INOCT, Zone 11) is the hyper-reflective region formed mainly by the ellipsoid component of the photoreceptors which are packed with the mitochondria where energy production and ATP production occurs, within the ellipsoid layer of the outer portion of the ISS of the photoreceptors. EZ was previously called as the photoreceptor IS/OS junction or IS/OS line which includes the connecting cilium and microtubules. In a normal fovea, EZ is located closer to ELM than RPE. ${ }^{48,9,15-20}$

The photoreceptor integrity line is the prominent bright band anterior to (or above) the RPE. This line was previously known as the connecting cilia, the junction of the ISS and OSS, and the IS ellipsoid. .1,22

The IZ (INOCT, Zone 13) is considered that it reflects the interdigitation of the apical processes of the RPE cells that encase part of OSs of the photoreceptors. It was previously called the cone OS tips and rod OS tips, and its differentiation from RPE layer is difficult for even healthy subjects. IZ lies anterior to the RPE complex and thus has been proposed to represent OS tips as its anatomic correlate. In the past, IZ has been attributed to Verhoeff's membrane. However, this nomenclature does not seem to be appropriate because the Verhoeff's membrane is not a true membrane and it is just an electron microscopy (EM) image corresponding to the band of the tight junction complexes. In addition, it was considered that IZ could not be equal to Verhoeff's membrane because IZ is located on the RPE layer. ${ }^{4-7,23,24}$

Verhoeff's membrane is an anatomical structure that surrounds the RPE cells, and it is also known as the tight junctional complexes between the RPE cells; it seems as a band on EM and it is sometimes called cone photoreceptor OS tips. ${ }^{23,24}$

The RPE/BM complex (INOCT, Zone 14) can be seen as two distinctive hyper-reflective bands separated by a hypo-reflective zone in highresolution images of SD-OCT. It was previously called single RPE line/ band. International nomenclature group agreed that these bands correspond with the RPE and the BM. In the past, this complex was also called RPE/BM/choriocapillaris (CC) complex. RPE/BM complex is thicker in the fovea compared to other retinal regions. ${ }^{3,4,9-15,25}$

The CC (INOCT, Zone 15) can seem as a thin layer of moderate reflectivity attributed to feeding arterioles to and draining venules from the CC in the inner choroid immediately below the RPE/BM complex. However, exact identification of these layers by current OCT devices in normal eyes is difficult. $34,9-15,25$

Sattler's layer (INOCT, Zone 16) is the thick layer of round- or oval-shaped hyper-reflective profiles with hypo-reflective cores corresponding to the small- or medium-sized oval vasculature to the Sattler's layer in midchoroid in SD-OCT Scans. ${ }^{3,49-15,25}$

Haller's layer (INOCT, Zone 17) is the thick layer of oval-shaped hyperreflective profiles with hypo-reflective cores in outer choroid corresponding to the larger outer oval vasculature to the Haller's layer,3,4,9-15,25

Choroidal-scleral junction (INOCT, Zone 18) may clearly be observed only with enhanced depth imaging mode of SD-OCT or swept source OCT. It is the zone at the outer choroid with a marked change in texture in which large circular or ovoid structures corresponding to the homogeneous region with variable reflectivity. The interest on the measurement of the choroidal thickness has been increased in recent years because it can be affected by various chorioretinal diseases. The clear detection and marking of this junction is very important for the correct measurement of choroidal thickness. ${ }^{3,4,25}$

In addition to the above-mentioned zones, in INOCT, Zone 2 attributes to the pre-retinal space; Zone 6 reflects the inner nuclear layer. Zone 12 reflects the OSs of the photoreceptors. Pre-retinal space can be observed as a hypo-reflective zone immediately above the ILM in the high-resolution OCT images in some healthy subjects. ${ }^{4}$

In conclusion, to use the standardised and proper OCT terms in current OCT terminology might provide standardisation in the definition of important pathologies and to understand better the pathogenesis and the effects of therapeutic applications for various macular diseases. $\square$ 
1. Arevalo JF, Lasave AF, Arias JD, et al., Clinical applications of optical coherence tomography in the posterior pole: The 2011 José Manuel Espino Lecture - Part I, Clin Ophthalmol, 2013:7:2165-79.

2. Gabriele ML, Wollstein G, Ishikawa $H$, et al., Optical coherence tomography: History, current status, and laboratory work, Invest ophthalmol Vis Sci, 2011;52:2425-36.

3. Spaide RF, Curcio CA, Anatomical correlates to the bands seen in the outer retina by optical coherence tomography: Literature review and model, Retina, 2011;31:1609-19.

4. Staurenghi G, Sadda S, Chakravarthy U, Spaide RF, International Nomenclature for Optical Coherence Tomography $($ IN*OCT) Panel, Proposed lexicon for anatomic landmarks in normal posterior segment spectral-domain optical coherence tomography: The $\mathrm{IN}{ }^{*} \mathrm{OCT}$ consensus, Ophthalmology, 2014;121:1572-8.

5. Turgut B, Demir T, The new landmarks, findings and signs in optical coherence tomography, New Front Ophthalmol, 2016;2:131-6.

6. Citırik M, IIhan C,, Teke MY, Optical coherence tomography (Optik Koherens Tomografi), Current Retina (Güncel Retina), 2017:1:58-68.

7. Koh A, Optical Coherence Tomography of the Outer Retinal Layers. In: Coscas G, Loewenstein A, Bandello F (eds), Optica Coherence Tomography, ESASO Course Series, Basel: Karge “ 2014;4:26-33.

8. Hogan MJ, Alvarado JA, Weddell JE, Histology of the Human Eye: An Atlas and Textbook, Philadelphia:W. B. Saunders,
1971;328-63

9. Drexler W, Cellular and functional optical coherence tomography of the human retina: The cogan lecture, Invest ophthalmol Vis Sci, 2007:48:5339-51.

10. Pinilla I Ruiz-Moreno JM, Cuenca N, Correlation of optical coherence tomography with retinal histology, Arch SOC ESP Oftalmol, 2012;87:275-7.

11. Drexler W, Morgner U, Ghanta RK, et al., Ultrahigh-resolution ophthalmic optical coherence tomography, Nat Med, 2001;7:502-7.

12. Fernandez EJ, Hermann B, Povazay B, et al., Ultrahigh resolution optical coherence tomography and pancorrection for cellular imaging of the living human retina, opt Express, 2008;16:11083-94.

13. Otani T, Yamaguchi Y, Kishi S, Improved visualization of Henle fiber layer by changing the measurement beam angle on optical coherence tomography Retina 2011:31:497-501.

14. Lujan BJ, Roorda A, Knighton RW, Carroll J, Revealing Henle's fiber layer using spectral domain optical coherence tomography, Invest Ophthalmol Vis Sci, 2011;52:1486-92.

15. Jonnal RS, Kocaoglu OP, Zawadzki RJ, et al., The cellular origins of the outer retinal bands in optical coherence tomography images, Invest Ophthalmol Vis Sci, 2014;55:7904-18.

6. Schaal KB, Rosenfeld PJ, The controversy of Band \#2, 2015. Available at: www.retina-specialist.com/article/thecontroversy-of-band-2\#sthash.FJSvgMyC.pdf (accessed 6 July 2017).

17. Srinivasan VJ, Monson BK, Wojtkowski M, et al., Characterization of outer retinal morphology with high-speed, ultrahighresolution optical coherence tomography, Invest Ophthalmol Vis Sci, 2008:49:1571-9.

18. Saxena S, Srivastav $\mathrm{K}$, Cheung $\mathrm{CM}$, et al., Photoreceptor inner segment ellipsoid band integrity on spectral domain optical coherence tomography, Clin Ophthalmol, 2014;8:2507-22.

19. Hoang QV, Linsenmeier RA, Chung CK, Curcio CA, Photoreceptor inner segments in monkey and human retina: Mitochondrial density, optics, and regional variation, Vis Neurosci, 2002;19:395-407.

20. Lu RW, Curcio CA, Zhang Y, et al., Investigation of the hype reflective inner/outer segment band in optical coherence tomography of living frog retina, J Biomed Opt, 2012;17:060504.

21. Sherman J, Epshtein D, The ABCS of OCT, Rev Optom, 2012:52-8. www.reviewofoptometry.com/CMSDocuments/2017/03/ ro0317i.pdf

22. Sherman J, Yannuzzi AL, Madonna RJ, et al., Photoreceptor Integrity Line: As Revealed by Spectral Domain OCT (eBook), New York: Lulu.com, 2008.

23. Verhoeff $F H, A$ hitherto undescribed membrane of the eye and its significance, R Lond Ophthalmic Hosp Rep, 1903;15:309-19.

24. Wolff $E$, The external limiting membrane of the retina and its relation to Verhoeff's membrane, Trans Ophthalmol Soc UK, 1950;60:61-7.

25. Huynh E, Chandrasekera E, Bukowska D, et al., Past, present, and future concepts of the choroidal scleral interface morphology on optical coherence tomography, Asia Pac Ophthalmol (Phila), 2017;6:94-103. 